\title{
Essai d'assainissement d'une zone infestée par Glossina fuscipes fuscipes Newst. en République Centrafricaine
}

\author{
par P. YVORE, J. DESROTOUR, J. LAURENT ef P. FINELLE
}

La trypanosomiase animale en République Centrafricaine constitue l'un des obstacles les plus importants à l'extension de l'élevage. Devant ce fait le service de l'Elevage de ce pays a envisagé différentes mesures tendant, soit à implanter du bétail tolérant dans les régions infestées par les glossines, soit à essayer d'assainir certaines zones de pâturages et à permettre ainsi le développement de l'élevage du zébu.

En 1960 une première campagne expérimentale a été réalisée sur la rivière Nié, dans la souspréfecture de Baboua près de la frontière de la République du Cameroun. La zone inféressée était infestée par Glossina fusca congolensis Newst. et Evans. Les résultats très satisfaisants obtenus au cours de cette campagne incitèrent à entreprendre un projet plus important intéressant une zone englobant le cours supérieur de la rivière Topia et ses affluents, dans le district de Carnot.

Ce nouveau projet a été financé par le Fond d'Aide et de Coopération, et a été réalisé au début de l'année 1962 en collaboration avec l'Institut d'Elevage et de Médecine vétérinaire des Pays tropicaux.

\section{LA HAUTE VALLÉE DE LA TOPIA}

\section{I) Raisons du choix de cette zone}

La zone intéressée par le projet se situe, environ, à $16^{\circ} 30^{\circ}$ de longitude Est et à $4^{\circ} 50^{\prime}$ à $5^{\circ}$ de latitude Nord. Cette région couvre tout le bassin supérieur de la Topia, affluent de la Lobaye, au nord de la route allant de Carnot à Boda, distante de 80 à 100 kilomètres de la première de ces villes.

Le bassin de la Topia est relativement bien isolé dans cette région. Mis à part une galerie

Rev. Elev. Méd. vét. Pays trop. 1962, 15, n 4. Reçu pour publication : Novembre 1962. forestière, le long de la rivière Bambouka, qui se trouve assez proche des sources de la Danou, il n'existe pas de galerie infestée à moins de 6 kilomètrcs de la zone traitée. Il était également possible d'isoler ce bassin en réalisant une coupure de galerie au niveau de la route CarnotBoda, sur la rivière Topia. En ce point la galerie forestière était relativement peu large et il était possible de la supprimer sur environ 2 kilomètres sans que cela nécessite des travaux trop importants. Cette coupure doit être augmentée prochainement en raison de l'installation d'un chantier minier. Ainsi l'isolement de la zone à traiter était relativement facile à réaliser. Ce premier fait a été l'une des raisons de son choix.

La deuxième raison réside dans le fait que l'assainissement de ce bassin permettrait l'utilisation d'environ 45.000 hectares de pâturages pour l'élevage du zébu. Il existe déjà, depuis quelques années, des troupeaux qui vivent dans cette région grâce à une surveillance sanitaire importante et à l'emploi de trypanocides. En cas de réussite du projet il serait, non seulement possible d'augmenter le nombre des animaux mais, également, de réaliser des économies de traitement et permettre aux infirmiers de ne plus être axés, presque exclusivement, sur le dépistage et le traitement de la trypanosomiase.

\section{2) Climat et végétation}

La haute vallée de la Topia se 'trouve située sur des formations sédimentaires anciennes, à base de grès, formant des sols sablonneux jaunâtres.

Cette région, d'après SILLANS, fait partie de la zone de sous-climat soudano-oubanguien, climat pré-forestier à pluviométrie annuelle moyenne de 1.400 à 1.500 millimètres. La suison sèche, de mi-novembre à avril, dure de 3 mois et demi à 4 mois selon les années. La saison des 

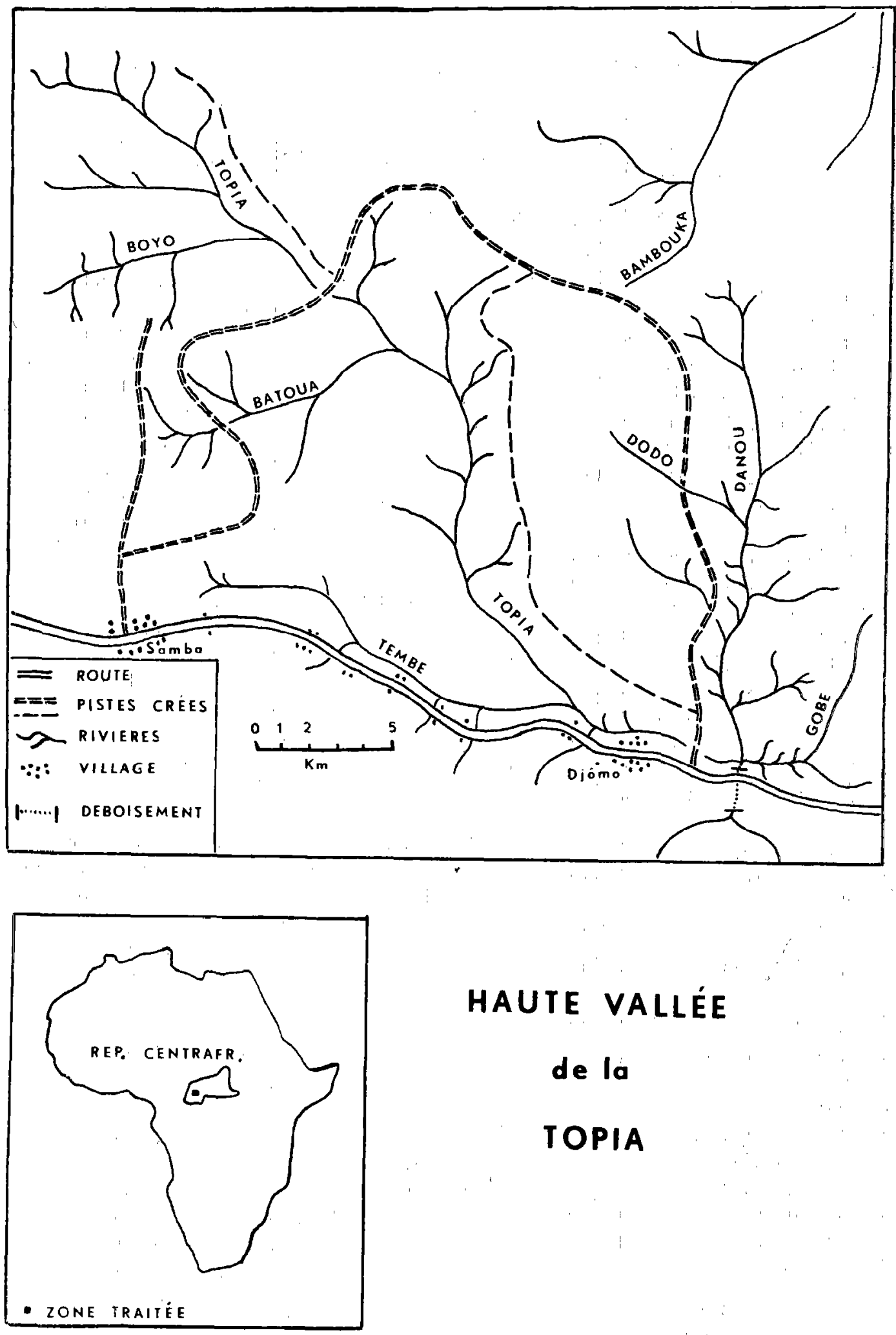

HAUTE VALLÉE

de la

TOPIA 
pluies est caractérisée par 3 périodes dont 2 de pluies plus ou moins fréquentes.

La région fait partie du district des savanes pré-forestières. Al'origine, elle était occupée par une forêt dense et humide de type oubanguien. Cette forêt a disparu presque complètement, détruite surtout par les populations locales. II en reste des vestiges constitués par les galeries forestières bordant les cours d'eau.

La savane est du type arbustive claire; savane à Terminalia et Albizzia avec, par place, des roneraies principalement dans l'Est de la zone intéressée par le projet.

Les cours d'eau sont bordés de galeries forestières. Celles-ci sont réduites, en certains points, à un « rideau d'arbres» mais elles peuvent atteindre, en d'autres, 500 mètres de large et plus. Ces galeries, assez denses, sont constituées par de grands arbres qu'entoure une végétation de lianes ef de buissons.

\section{3) Ełude entomologique}

En saison des pluies toutes les galeries sont infestées par Glossina fuscipes fuscipes Newst., seule glossine capturée jusqu'à maintenant dans cette région.

Cette mouche est présente toute l'année mais. durant la saison sèche, elle se concentre en certains points où la galerie est plus épaisse et plus ou moins marécageuse, le long des rivières les plus importantes. Les captures, à ce moment de l'année. sont faibles et un captureur ne peut trouver plus de 10 à 15 de ces mouches en une matinée.

On admet généralement que cette espèce s'éloigne assez peu du bord de la rivière mais ici, étant donné que les galeries forestières sont peu larges, souvent marécageuses, et que l'on peut penser que pour trouver sa nourriture la tsétsé doit, nécessairement, se rendre dans des régions plus dégagées, on peut considérer que la totalité de la galerie est infestée. Pourtant il nous a été souvent difficile de capturer des glossines dans la bordure des galeries.

Au cours de la saison des pluies 1961, nous avons pu observer 155 mouches au repos ou attendant un hôte. Ce dernier cas serait le plus général car très peu de ces glossines étaient gorgées au moment ou elles ont été capturées.

Parmi ces 155 mouches tsétsé nous avons dénombré 86 mâles $(54,8$ p. 100$)$ et 69 femelles $(45,8$ p. 100). Nous avons noté la hauteur à laquelle la glossine était posée au-dessus du sol et la nature de son support : tronc, branches ou feuilles.

Le nombre des captures est relativement restreint mais il peut néanmoins donner une indication sur les habitudes de repos de Glossina fuscipes. Nous donnons au tableau et graphique I les résultats obtenus. On peut constater que 94 p. 100 des mouches tsétsé étaient posées entre 1 mètre et 1,50 mètre au-dessus du sol. Aucune glossine n'a été capturée à plus de 1,60 mètre. Enfin les mâles et les femelles semblent avoir le même comportement.

L'observation de la nature du support a porté sur un nombre plus élevé de mouches tsétsé (207) et a donné les résultats suivants :

- 93 Glossina fuscipes fuscipes (44,9 p. 100) étaient posées sur le tronc de l'arbre.

- 90 Glossina fuscipes fuscipes (43,4 p. 100) étaient posées sur des feuilles.

- 34 Glossina fuscipes fuscipes (11, 7 p. 100) étaient posées sur des branches basses.

En outre it he semble pas que le choix du lieu de repos soit en rapport avec l'espèce botanique de l'arbre. En conséquence, il a été décidé d'appliquer les pulvérisations sur toute la végétation, jusqu'à une hauteur d'environ 2 mètres audessus du sol, ce qui semble suffisant en regard des observations précédentes.

En ce qui concerne l'activité de Glossina fuscipes, ITARD (comm. pers.) a constaté que, si les conditions atmosphériques sont favorables, cette tsétsé peut être active dès 7 heures du matin et qu'il est encore possible d'en capturer après 17 heures. Cette espèce pourrait, d'après WILSON (1953), franchir des barrières de débroussaillements de plus de $5 \mathrm{~km}$ et voyager, le long des rivières, sur des distances de 16 à 20 kilomètres.

La durée de pupaison serait en moyenne de 25 à 39 jours suivant le sexe et la saison (MAILLOT 1958).

\section{RÉALISATION DU PROJET}

\section{I) Bases techniques}

Débroussaillement, destruction du gibier, pulvérisation d'insecticides par avion ou du sol 


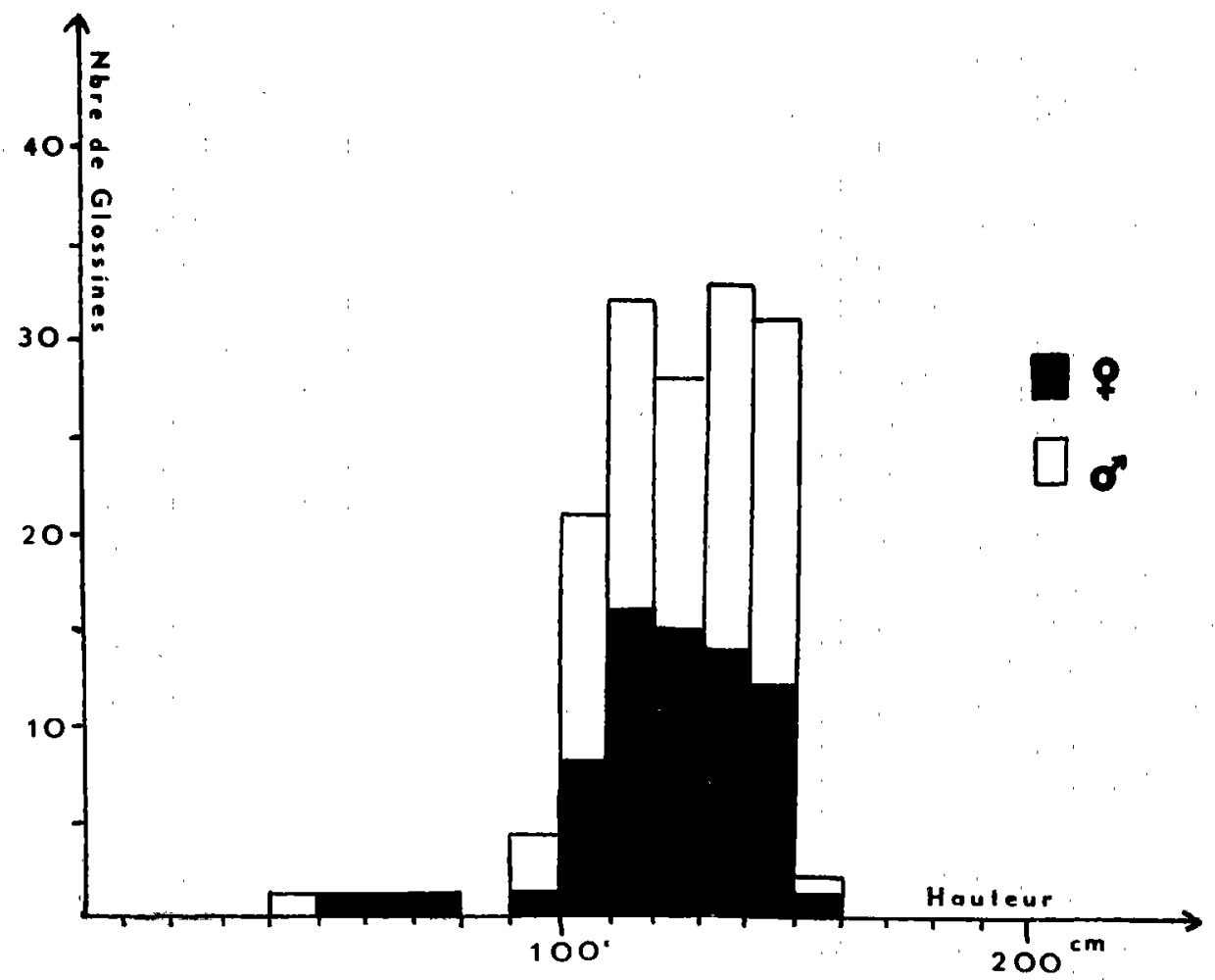

Graphique 1.

\begin{tabular}{|c|c|c|c|c|}
\hline \multicolumn{5}{|c|}{$\frac{\text { TABLEAU I }}{\text { Newst - Glossina fuscipes fuscipes }}$} \\
\hline $\begin{array}{l}\text { Hauteur } \\
(\mathrm{cm} .)\end{array}$ & Femelles & Males & Total & \\
\hline $0-10$ & 0 & 0 & 0 & \\
\hline $10-20$ & 0 & 0 & 0 & \\
\hline $20-30$ & 0 & 0 & 0 & \\
\hline $30-40$ & 0 & 0 & 0 & \\
\hline $40-50$ & 0 & 1 & 1 & \\
\hline $50-60$ & 1 & 0 & 1 & \\
\hline $60-10$ & 1 & 0 & 0 & ' \\
\hline $70-80$ & 1 & 0 & 1 & \\
\hline $80-90$ & 0 & 0 & 0 & \\
\hline $90-100$ & 1 & 3 & 4 & \\
\hline $100-110$ & 8 & 15 & 21 & \\
\hline $110-120$ & 16 & 16 & 52 & \\
\hline $120-130$ & 15. & $1 j$ & 28 & \\
\hline $130-140$ & 14 & 19 & 35 & \\
\hline $140-150$ & 12 & 19 & 31 & \\
\hline $150-160$ & 1 & 1 & 2 & \\
\hline
\end{tabular}


ont été tour à tour essayés, avec plus ou moins de succès, en vue de détruire les tsétsé infestant une zone. La seule méthode qui semble intéressante est la dernière. WILSON, au Kénya, DAVIES, MCLENNAN ef KIRKBY, en Nigéria, et MOUCHET, DELAS et YVORE en Républiques du Tchad et du Cameroun, ont obtenu d'excellents résultats en employant comme insecticide le D. D. T. ou la dieldrin. Enfin, en République Centrafricaine, cette méthode semble avoir permis de détruire complètement Glossine fusca congolensis Newst. et Evans sur la rivière Nié (FINELLE, DESROTOUR et YVORÉ 1962).

II est nécessaire, pour la réussite d'un tel projet, de réunir plusieurs conditions: Traiter tous les lieux de repos des glossines présentes. employer un insecticide à rémanence suffisante pour permettre d'atteindre tous les imagos éclos après la fin du traitement, enfin éviter la réinfestation en isolant parfaitement la région traitée.

Comme nous l'avons vu précédemment, la zone intéressée par le projet était relativement facile à isoler des autres régions infestées. II suffisait de réaliser une coupure de galerie, sur la Topia, au niveau de la route Carnot-Boda. Cette coupure a été pratiquée sur 2 kilomètres. II est possible qu'elle soit insuffisante pour protéger la région traitée si, comme l'affirme WILSON, Glossina fuscipes peut franchir des barrières de 5 . kilomètres. Des contrôles devront avoir lieu régulièrement au niveau de la coupure et, également, au niveau des sources de la Danou proches des galeries infestées bordant la Bambouka. Au cas où l'on constaterait une réinfestation dans ces zones il pourrait être possible de renforcer la protection par des pulvérisations périodiques, limitées, d'insecticides, créant ainsi une barrière chimique.

La durée de pupaison a été évaluée par MAILLOT à un maximum de 39 jours. Il est donc nécessaire que l'effet résiduel de l'insecticide déposé sur la végétation persiste plus d'un mois. La dieldrin, insecticide de contact, a une rémonence suffisante pour couvrir cette période. Néanmoins cet insecticide peut être emporté par les pluies, c'est pourquoi les pulvérisations doivent être réalisées durant la saison sèche et terminées un certain temps avant les premières pluies. Par contre il est possible de les commencer dès le début de la saison sèche : toutes les galeries devant être traitées sans tenir compte de la répartition plus limitée durant cette saison.

Toute la végétation, en dessous d'une hauteur de 2 mètres, doit recevoir des pulvérisations puisqu'il ne semble pas qu'il soit possible d'appliquer un traitement sélectif sur certaines espèces botaniques.

\section{2) Exécution des travaux}

\section{a) Phase préparatoire}

Après une reconnaissance géographique $\mathrm{mi}$ nutieuse de la zone à traiter, reconnaissance facilitée par l'emploi de photographies aériennes, une équipe entomologique a étudié l'infestation de la zone et certains aspects de l'écologie de Glossina fuscipes intéressants pour la réalisation du projet. Pendant ce temps, des équipes de manœuvres créaient des pistes ct réalisaient la barrière de débroussaillement sur la Topia. Une piste principale a été créée. Elle doit servir par la suite au contrôle sanitaire de la zone d'élevage. En outre, des pistes très sommaires, ne devant être employées que durant l'opération, ont été réalisées le long des galeries forestières pour permettre d'amener le personnel sur son lieu de travail.

\section{b) Phase d'exécution}

Les pulvérisations ont débuté le 18 décembre 1961 et se sont terminées le 19 février 1962, soit une durée de 2 mois.

\section{L'insecticide.}

Nous avons utilisé du « Dieldrex CE 20 », liquide concentré, émulsifiable, contenant 20 p. 100 de dieldrin. Cette présentation nous a donné satisfaction. Pourtant il semble préférable d'employer la poudre movillable à 50 p. 100 de produits actifs. Cette forme, que l'un de nous a employée au cours d'une autre campagne, semble d'un emploi plus facile et moins délicat.

Le «Diedrex» était dilué au 1/10 au moment de l'emploi, de manière à obtenir une concentration à 2 p. 100 de produit actif. En tout, un peu plus de 6.000 litres furent utilisés.

Les pulvérisateurs.

Nous avons employé 20 pulvérisateurs « Galéazzi » à dos, à pression préalable, d'une capacité utile de 12 litres. Ces appareils nous ont donné toute satisfaction. lis ont l'avantage d'être relativement légers, ce qui est important en raison des conditions, souvent très dures, de travail : 
pulvérisations en galeries forestières souvent très touffues et plus ou moins marécageuses.

\section{Personnel.}

Une partie du personnel ayant déjà participé à une campagne similaire, le problème de sa formation a été simplifié. Il est important de bien montrer aux pulvérisateurs le travail à exécuter et ils doivent être constamment surveillés par les chefs d'équipe vérifiant si la totalité de la végétation a été traitée correctement.

Les équipes de pulvérisation étaient, en général, constituées de 7 hommes : 3 pulvérisateurs, 3 mancuvres facilitant la progression des précédents en créant des passages à la matchette, et un mancuvre portant 20 litres de «Dieldrex» et un seau à mélange.

Selon l'importance de la galerie, le nombre des équipes travaillant de front était modifié. En général il suffisait'd'une équipe de chaque côté de la rivière.

Environ 250 kilomètres de galeries furent ainsi traités. En moyenne on employa 24 litres de Dieldrex au kilomètre et on traita 4 kilomètres par jour environ.

\section{c) Phase de contrôle}

Une équipe entomologique est restée sur la zone traitée. Cinq points de contrôle avaient été choisis en raison de l'importance de leur infesfation. Ces points sont régulièrement visités. En outre, l'équipe entomologique a pour mission de surveiller particulièrement les abords de la coupure, du côté traité, et les sources de la Danou proches de la galerie longeant la Bambouka.

L'équipe entomologique doit séjourner au moins un an en permanence sur la zone. Par la suite on envisagera des contrôles moins réguliers.

\section{PRIX DE REVIENT}

Le prix de revient total de cette opération a été de 5.670 .500 francs. CFA qui se répartissent comme suit :

- Main-d'œuvre ......... $1.450 .000 \mathrm{fr}$. CFA

- Insecticide ..............

- Appareils de pulvérisation ................

- Entretien véhicules, essence ..............

- Petit matériel, divers....

\section{$2.772 .712 \mathrm{fr}$. CFA}

$292.328 \mathrm{fr}$. CFA

$655.460 \mathrm{fr}$. CFA

$500.000 \mathrm{fr}$. CFA
Dans ce prix de revient ne sont pas compris les salaires du personnel affecté à d'autres services (entomologistes, contrôleurs d'élevage, auxiliaires d'entomologie, chauffeurs) ni l'amortissement, ou l'achat de véhicules.

Si l'on considère que la réalisation de ce projet peut permettre d'assainir 45.000 hectares, le prix de revient à l'hectare est d'environ $125 \mathrm{fr}$. CFA.

\section{RÉSULTATS}

Il est encore prématuré de donner les résultats de cette campagne. Néanmoins ceux-ci, après une saison des pluies, semblent encourageants.

Huit jours après la fin du traitement d'une zone infestée il n'a plus été possible de trouver de Glassina fuscipes fuscipes Newst. Les glossines par contre, étaient toujours présentes dans les galeries non traitées.

Pourtant une mouche fut observée et capturée le 10 mars au niveau d'une galerie, particulièrement dense et marécageuse située a peu près au milieu de la zone, sur la rivière Topia. Cette galerie avait"été traitée aux environs du 12 février. La glossine capturée avait tous les caractères d'une mouche «ténérale » ${ }^{*}$ ) (nouvellement éclose): Il fut décidé de' continuer la surveillance sans appliquer de nouveau traitement. Depuis aucune autre mouche n'a été observée. Il est probable que la glossine capturée le 10 mars provenait d'une pupe déposée avant le traitement de la zone et pas encore atteinte par l'insecticide.

\section{CONCLUSIONS}

Le principe de la lutte contre les glossines est maintenant bien connu. A l'heure actuelle trois campagnes ont été réalisées en Afrique d'expression française. La campagne de la haute vallée de la Topia est la dernière en date. II est encore trop tôt pour pouvoir considérer la zone comme assainie mais, après presque 1 an et

(*) Nous avons employé le terme «ténéralè », à l'exemple des anglais qui parlent de «teneral flies » pour désigner des imagos nouvellement éclos qui possèdent encore une cuticule molle et sur lesquels, il est encore possible de faire saillir le ptilinum. "Ténérale » venant du latin « tener », nous pensons qu'il est possible de l'employer en français pour rendre ce caractère propre aux très jeunes imagos. BUXTON, dans « The natural history of tsé-tsé flies » donne une définition de ce terme. 
après une saison de pluies, les premiers résultats sont très encourageants.

Un autre projet, de plus grande importance est à l'étude dans l'Est de la République Centrafricaine. Il sera réalisé en fonction des résultats obtenus dans la région de la Topia.

Centre de recherches sur les trypanosomiases onimales de Bouor.

- Service de l'Elevage de la République Centrafricaine.

\section{RÉSUMÉ}

Une campagne de lutte contre Glossina fuscipes fuscipes Newst. par pulvérisation d'insecticide à partir du sol a été réalisée en République Centrafricaine au début de l'année 1962.

L'insecticide employé fut le dieldrin en émulsion à 2 p. 100. de produit actif. Toute la végétation a été traitée jusqu'à une hauteur d'environ 2 mètres.

Jusqu'ici aucune glossine n'a éfé capturée dans cette région depuis la fin du traitement, à part une mouche « ténérale » moins d'un mois après le traitement de la zone où elle a été capturée.

Coût : 125 francs CFA par hectare de pâturage assaini.

\section{SUMMARY}

Tsetse eradication trial in a zone infested with $G$. fuscipes in the Central African Republic

A control trial against $G$. fuscipes fuscipes Newst. by spraying from the ground was carried out in the Central African Republic at the beginning of 1962.

The insecticide employed was dieldrin in an emulsion of 2 per Cent of the active principle. All vegetation to a height of about 6 feet was treated.

Up to date no glossina has been captured in this region since the end of the period of treatment other than one teneral fly which was taken under one month after the area in which it was found had been treated.

The cost was about 1.4 shillings per acre.

\section{RESUMEN}

Ensayo de saneamiento en una zona infestada por Glossina fuscipes, en la República Centroafricana

A principios del año 1962, se ha realizado en la República Centroafricana una campaña de lucha contra la Glossina fuscipes fuscipes Newst.. por pulverización de producto insecticida a partir del suelo.

El insecticida empleado ha sido el Dieldrin, en emulsión de 2 p. 100 de producto activo. Toda la vegetación ha quedado tratada hasta una altura de dos metros.

Hasta la fecha, no se ha capturado ninguna Glossina en esta región desde el final del tratamiento. salvo una mosca «teneral » menos de un mes después del tratamiento de la zona en que ha sidocapturada.

Coste : 125 francos CFA por hectrâea de terreno de pasto tratado.

\section{BIBLIOGRAPHIE}

AUBREVILLE (A.) - Flore forestière soudanoguinéenne (A. O. F. Cameroun A. E. F.). Soc. Ed. Geogr. Mar. Col. Paris 1950.

BARROS MACHADO (A. de) - Révision systématique des glossines du groupe palpalis (Diptera). Publ. Cult. Co. Diam. Ang., Lisboa 1957, 22, 82-94.
BUXTON (P. A.) - The natural history of tsétsé flies. Lewis \& Co, London, 1955.

DAVIES (J. B.) - An attempt to eradicate Glossina palpalis R. D. and Glossina tachinoïdes Westw. from riverine vegetation in Benue Province, Northern Nigéria, by spraying with D. D. T. Bull. ent. Res. 1959, 49, 427-436. 
DAVIES (J. B.) - Use of Dieldrin in the control of Glossina palpalis (R. D.) in Nigéria. Nature 1960, 187, 84-85.

DAVIES (J. B.) et BLASDALE (P.) - The eradication of Glossina morsitans submorsitons Newst. and Glossina tachinoïdes Westw. in part of a river flood plain in Northern $\mathrm{Ni}$ géria by chemical means. Bull. ent. Res., 1960 , 5I, 265-7.70.

FAIRCLOUGH (R.) et THOMSON (W. E. F.) The effect of insecticidal spraying against Glossino palpalis fuscipes Newst. in the Nyando River basin of Kenya. East Afr. Agr. J., 1958, 23, 186-189.

FINELLE (P.) - Les trypanosomiases bovines dans l'Ouest de l'Oubangui-Chari. Essais de traitement par le Bérénil. Rev. Elev. Méd. vét. Pays trop., 1957, 10, 231-247.

FINELLE (P.) DESROTOUR (J.) et YVORÉ (P.) Essai de lutte contre Glossina fusca par pulvérisation de dieldrin en République Centrafricaine. Rev. Elev. Méd. vét. Pavs. trop., 1962, 3.

HOCKING (K. S.) - Discriminative insecticide spraying against Glossina morsitans Westw. Bull. ent. Res., 1961, 52, 17-22.

KIRKBY (W.W.) et BLASDALE (P.) - The eradication of Glossina morsitans submorsitans
Newst. and Glossina fachinoïdes Westw. in part of a river flood plain in Northern $\mathrm{Ni}$ géria by chemical means. Bull. ent. Res., 1960, 5I, 253-263.

MCLENNAN (K. J. R.) ef KIRKBY (W. W.) The eradication of Glossina morsitans submorsitans Newst. in part of a river flood plain in Northern Nigéria by chemical means. Bull. ent. Res., 1958, 49, 123-131.

MAILLOT (L.) - Elevage de Glossina fuscipes quanzensis Pires à Brazzaville. Bull. I. E. C: 1958, I5-16, 85-90.

MOUCHET (J.) DELAS (J.) et YVORÉ (P.) - La campagne expérimentale de lutte contre Glossina tachinoïdes West. à Logone-Birni. Bull. Soc. Path. exot., 1961, 54, 875-892.

SILLANS (R.) - Les savanes de l'Afrique centrale. Encyclopédie biologique. Edit. Lechevalier, Paris 1958.

TROCHAIN (L.) - Accord interafricain sur la définition des types de végétation de l'Afrique. Bull. I. E. C. 1957, I3-|4, 53-93.

WILSON (S. G.) - The constral of Glossina palpalis fuscipes Westw. in Kenya colony. Bull. ent. Res., 1953, 44, 711-728.

I. E. M. V.P.T. - Centre de Recherches de Bouar. Rapport annuel 1961.

Service de l'Elevage de la R. C. A.: Rapports annuels. 